\title{
THE EXISTENCE OF GORENSTEIN FLAT COVERS
}

\author{
EDGAR E. ENOCHS, OVERTOUN M. G. JENDA and J. A. LOPEZ-RAMOS*
}

\begin{abstract}
We prove that all left modules over a right coherent ring have Gorenstein flat covers.
\end{abstract}

\section{Introduction and Preliminaries}

The notion of a cotorsion theory was introduced by L. Salce in [14] and its importance in homological algebra has been shown by its use in the proof of the "flat cover conjecture". The existence of flat covers for every module over any ring was conjectured by E. Enochs ([6], 1981). When P. Eklof and J. Trlifaj [5] showed the connection between cotorsion theories which are cogenerated by a set and cotorsion theories with enough injectives and projectives, the positive answer to that conjecture seemed near. In fact this was the case and the conjecture has now been settled by L. Bican, R. El Bashir and E. Enochs [3].

More recently, M. Hovey [13] has shown that there is an intimate connection between Quillen's model of structure on abelian categories and complete cotorsion theories with respect to some proper class of short exact sequences. Hovey gave examples of such model structures using the cotorsion theories which appear in the context of the so called Gorenstein modules (these involve generalizations of notions introduced by Auslander [1] in connections with his study of G-dimensions). It is now well known that over Gorenstein rings, the classes of Gorenstein injective and projective modules form part of two corresponding complete cotorsion theories (see [11] for example). It is an intriguing question (and apparently an open one) whether this result has an extension to more general rings. An even more basic question is whether every module has a Gorenstein projective cover. Even if the ring is commutative and noetherian it is not known whether this is the case. But in this article we will show that if our ring $R$ is right coherent then every left $R$-module has a

\footnotetext{
* The second author acknowledges the support of an EPSRC research studentship. Several figures were drawn with the Liverpool Surfaces Modelling Package (LSMP); see .

Received September 16, 2002; in revised form September 5, 2003.
} 
Gorenstein flat precover. Then since the class of Gorenstein modules is closed under direct limits we get further that each of these modules has a Gorenstein flat cover. The existence of Gorenstein flat covers was first proved for modules over Gorenstein rings (see [9]) and then their existence is proved for certain modules over any local Cohen-Macaulay ring admitting a dualizing module [10]. The latter result has been extended by H. Holm in [12, Proposition 3.2.4].

We introduce the concept of a perfect cotorsion theory. We define this as a cotorsion theory $(\mathscr{L}, \mathscr{C})$ such that every $R$-module has an $\mathscr{L}$-cover and a $\mathscr{C}$-envelope. Immediate examples are ( $R$-Mod, Inj) and (Flat, Cot) where "Inj", "Flat" and "Cot" denote the classes of injective, flat and cotorsion $R$ modules respectively. Using the result mentioned above we show that the class of Gorenstein flat (left) modules along with its right orthogonal class form a perfect cotorsion theory over any right coherent ring. We also consider the class of weakly Gorenstein flat modules (see 3 for the definition) and obtain a relative version of the "flat cover conjecture".

Throughout this paper, $R$ will denote an associative ring and $R$-module will mean left $R$-module. $R$-Mod will denote the category of left $R$-modules and given any $R$-module $M, E(M)$ will be the injective envelope of $M$.

Given a class of $R$-modules $\mathscr{L}$, we will denote by $\mathscr{L}^{\perp}$ (respectively ${ }^{\perp} \mathscr{L}$ ) the (right orthogonal) class of $R$-modules $M$ such that $\operatorname{Ext}_{R}^{1}(L, M)=0$ (respectively $\operatorname{Ext}_{R}^{1}(M, L)=0$ ) for every $L \in \mathscr{L}$. Now using this notation, we recall that a pair of classes of $R$-modules $(\mathscr{L}, \mathscr{C})$ is said to be a cotorsion theory if $\mathscr{L}^{\perp}=\mathscr{C}$ and ${ }^{\perp} \mathscr{C}=\mathscr{L}$.

Also recall that a cotorsion theory $(\mathscr{L}, \mathscr{C})$ is said to be complete (cf. [13]) if it has enough injectives and projectives, that is, for every $R$-module $M$ there are exact sequences $0 \rightarrow M \rightarrow C \rightarrow L \rightarrow 0$ and $0 \rightarrow \bar{C} \rightarrow \bar{L} \rightarrow M \rightarrow 0$ respectively with $C, \bar{C} \in \mathscr{C}$ and $L, \bar{L} \in \mathscr{L}$. This concept is intrinsically related to the existence of $\mathscr{C}$-preenvelopes and $\mathscr{L}$-precovers, respectively, for every $R$-module. We recall from [6] that given a class of $R$-modules $\mathscr{L}$, a morphism $\varphi: L \rightarrow M$ where $L \in \mathscr{L}$ is called an $\mathscr{L}$-precover of $M$ if for any morphism $\psi: L^{\prime} \rightarrow M$ we get a commutative diagram

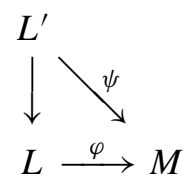

In the case that $L^{\prime}=L$ and $\psi=\varphi$ and we can only complete the preceding diagram by automorphisms, we say that $\varphi: L \rightarrow M$ is an $\mathscr{L}$-cover. $\mathscr{L}$ preenvelope and $\mathscr{L}$-envelope are defined dually.

If $0 \rightarrow M \rightarrow C \rightarrow L \rightarrow 0$ and $0 \rightarrow \bar{C} \rightarrow \bar{L} \rightarrow M \rightarrow 0$ are as above, then for $C^{\prime} \in \mathscr{C}, \operatorname{Hom}\left(C, C^{\prime}\right) \rightarrow \operatorname{Hom}\left(M, C^{\prime}\right) \rightarrow \operatorname{Ext}^{1}\left(L, C^{\prime}\right)=0$ is exact 
and so $M \rightarrow C$ is a $\mathscr{C}$-preenvelope. Similarly $\bar{L} \rightarrow M$ is an $\mathscr{L}$-precover.

\section{Hereditary and perfect cotorsion theories}

In this section we give properties of cotorsion theories related to the existence of covers and envelopes. Clearly much of what we say has meaning and is valid in any abelian category with enough injectives and projectives.

Definition 1.1. We will say that the cotorsion theory $(\mathscr{L}, \mathscr{C})$ is hereditary if whenever $0 \rightarrow L^{\prime} \rightarrow L \rightarrow L^{\prime \prime} \rightarrow 0$ is exact with $L, L^{\prime \prime} \in \mathscr{L}$ then $L^{\prime}$ is also in $\mathscr{L}$.

The following result shows that in a hereditary cotorsion theory $(\mathscr{L}, \mathscr{C})$, the classes $\mathscr{L}$ and $\mathscr{C}$ satisfy dual properties and that in this case the "orthogonality relation" holds for each functor $\mathrm{Ext}^{n}$ with $n \geq 1$.

Proposition 1.2. Let $(\mathscr{L}, \mathscr{C})$ be a cotorsion theory. Then the following are equivalent:

(i) $(\mathscr{L}, \mathscr{C})$ is hereditary.

(ii) If $0 \rightarrow C^{\prime} \rightarrow C \rightarrow C^{\prime \prime} \rightarrow 0$ is exact with $C^{\prime}, C \in \mathscr{C}$ then $C^{\prime \prime}$ is also in $\mathscr{C}$.

(iii) $\operatorname{Ext}_{R}^{i}(L, C)=0$ for all $i \geq 1$ and all $L \in \mathscr{L}$ and $C \in \mathscr{C}$.

Proof. (i) $\Rightarrow$ (iii) Let $L \in \mathscr{L}$ and $0 \rightarrow L^{\prime} \rightarrow P \rightarrow L \rightarrow 0$ be an exact sequence with $P$ projective. Then $L^{\prime} \in \mathscr{L}$ by hypothesis since $P \in \mathscr{L}$. So if $C \in \mathscr{C}$, then the result follows by the long exact sequence of Ext associated to the preceding short exact sequence.

(iii) $\Rightarrow$ (i) Let $C \in \mathscr{C}$ and $0 \rightarrow L^{\prime} \rightarrow L \rightarrow L^{\prime \prime} \rightarrow 0$ be exact with $L, L^{\prime \prime} \in \mathscr{L}$. Then the result follows from the exact sequence

$$
\cdots \rightarrow \operatorname{Ext}_{R}^{1}(L, C) \rightarrow \operatorname{Ext}_{R}^{1}\left(L^{\prime}, C\right) \rightarrow \operatorname{Ext}_{R}^{2}\left(L^{\prime \prime}, C\right) \rightarrow \cdots
$$

(iii) $\Rightarrow$ (ii) Let $0 \rightarrow C^{\prime} \rightarrow C \rightarrow C^{\prime \prime} \rightarrow 0$ be exact with $C^{\prime}, C \in \mathscr{C}$. If $L \in \mathscr{L}$, then by the exactness of

$$
\cdots \rightarrow \operatorname{Ext}_{R}^{1}(L, C) \rightarrow \operatorname{Ext}_{R}^{1}\left(L, C^{\prime \prime}\right) \rightarrow \operatorname{Ext}_{R}^{2}\left(L, C^{\prime}\right) \rightarrow \cdots
$$

we get that $\operatorname{Ext}_{R}^{1}\left(L, C^{\prime \prime}\right)=0$ for all $L \in \mathscr{L}$. So $C^{\prime \prime} \in \mathscr{L}^{\perp}=\mathscr{C}$.

(ii) $\Rightarrow$ (iii) follows by a dual argument to the proof of (i) $\Rightarrow$ (iii) above by considering an exact sequence $0 \rightarrow C \rightarrow E \rightarrow C^{\prime} \rightarrow 0$ with $E$ injective.

Definition 1.3. A cotorsion theory $(\mathscr{L}, \mathscr{C})$ is said to be perfect if every module has an $\mathscr{L}$-cover and a $\mathscr{C}$-envelope. 
TheOREM 1.4. Let $(\mathscr{L}, \mathscr{C})$ be a hereditary cotorsion theory. Then the following are equivalent:

(i) $(\mathscr{L}, \mathscr{C})$ is perfect.

(ii) Every $R$-module has a $\mathscr{C}$-envelope and every $C \in \mathscr{C}$ has an $\mathscr{L}$-cover.

(iii) Every $R$-module has an $\mathscr{L}$-cover and every $L \in \mathscr{L}$ has a $\mathscr{C}$-envelope.

Proof. By definition it is clear that (i) $\Rightarrow$ (ii), (iii). We will show (iii) $\Rightarrow$ (i) and the proof of (ii) $\Rightarrow$ (i) is dual to this.

Let $M$ be an $R$-module and let $\varphi: L \rightarrow M$ be an $\mathscr{L}$-cover. Then $\varphi$ is an epimorphism since $\mathscr{L}$ contains projective modules, and since $\mathscr{L}$ is closed under extensions it follows that $G=\operatorname{Ker}(\varphi) \in \mathscr{C}$ by the Wakamatsu Lemma (cf. [11, Corollary 7.2.3]). Now let $L \rightarrow H$ be a $\mathscr{C}$-envelope. Similarly we get that $0 \rightarrow L \rightarrow H \rightarrow N \rightarrow 0$ is exact with $N \in \mathscr{L}$. Then consider the pushout diagram

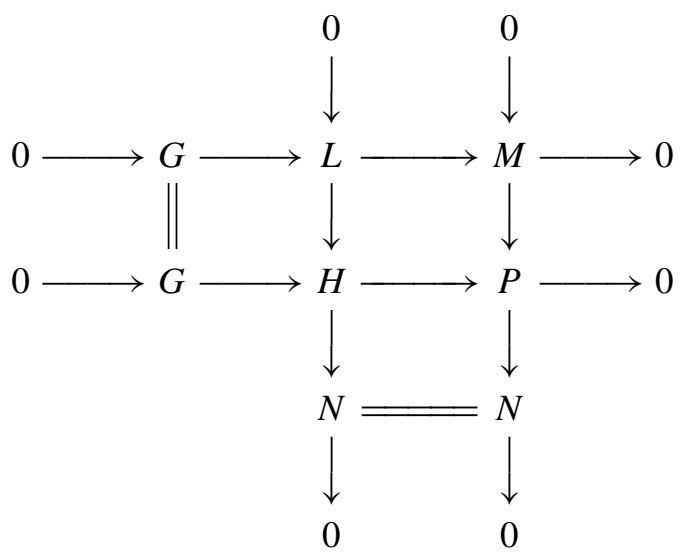

Since $(\mathscr{L}, \mathscr{C})$ is hereditary, $P \in \mathscr{C}$ by the proposition above, and since $N \in \mathscr{L}$ we get that $M \rightarrow P$ is a $\mathscr{C}$-preenvelope. We now show that it in fact a $\mathscr{C}$ envelope.

We first observe that the square

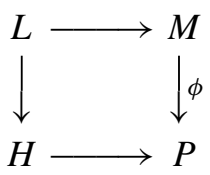

is not only a pushout diagram, but is also a pullback diagram. And $H \rightarrow P$ is a $\mathscr{L}$-precover whose kernel is in $\mathscr{C}$. This follows from the fact that $G \in \mathscr{C}=\mathscr{L}^{\perp}$ and that $H \in \mathscr{L}$ since $0 \rightarrow L \rightarrow H \rightarrow N \rightarrow 0$ is exact with $L, N \in \mathscr{L}$. 
Now we must argue that any morphism $f: P \rightarrow P$ such that $f \circ \phi=\phi$ is an automorphism of $P$. Since $H \rightarrow P$ is an $\mathscr{L}$-precover, the diagram

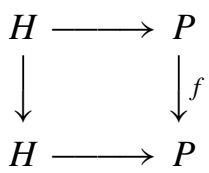

can be completed to a commutative diagram. Then the morphism $H \rightarrow H$ along with $f: P \rightarrow P$ gives a morphism of the diagram

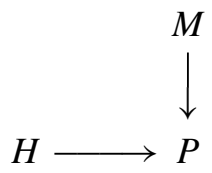

to itself, which is the identity morphism on $M$ and is $f$ on $P$. Now since (*) is a pullback we get a morphism of (*) to itself which is the identity on $M$ and which is $f$ on $P$. Since $L \rightarrow M$ is an $\mathscr{L}$-cover, the morphism $L \rightarrow L$ is an automorphism. Then since $L \rightarrow H$ is a $\mathscr{C}$-envelope, the morphism $H \rightarrow H$ is also an automorphism. But now, since $(*)$ is a pushout and since our morphism of this square to itself is an isomorphism on $L, M$ and $H$ we get that it is also an isomorphism on $P$ and so $f$ is an automorphism as we desired.

\section{Gorenstein flat covers over coherent rings}

The aim of this section is to prove the existence of Gorenstein flat covers for every module over a right coherent ring. So throughout this section $R$ will denote a right coherent ring. We recall from [8] that an $R$-module $M$ is said to be Gorenstein flat if and only if there exists an exact sequence in $R$-Mod

$$
\cdots \rightarrow F_{1} \rightarrow F_{0} \rightarrow F_{-1} \rightarrow F_{-2} \rightarrow \cdots
$$

of flat $R$-modules such that $M=\operatorname{Ker}\left(F_{-1} \rightarrow F_{-2}\right)$ and which remains exact whenever $E \otimes_{R}-$ is applied for any injective right $R$-module $E$.

We also recall from [7, Proposition 3.3] that over right coherent rings we can define right derived functors of $-\otimes_{R}-$ by using injective resolutions and right Flat-resolutions (see [7] or [11]) in the first and second variables respectively. These new derived functors are denoted by $\operatorname{Tor}^{n}$. We also note that there exists a natural morphism

$$
M \otimes_{R} N \rightarrow \operatorname{Tor}^{0}(M, N)
$$

Since $R$ is a right coherent ring, then every left $R$-module has a flat preenvelope by [11, Proposition 6.5.1]. Now, by [11, Corollary 6.4.4], if $M_{i} \rightarrow F_{i}$ is a flat 
preenvelope for every $M_{i} i \in I$ then $\oplus M_{i} \rightarrow \oplus F_{i}$ (arbitrary direct sum) is a flat preenvelope. In this way if we consider $\left\{\mathscr{F}^{i}\right\}$ a family of flat right resolutions of the family of left modules $\left\{M_{i}: i \in I\right\}$ and we consider the direct sum of these complexes, then this new complex is a flat right resolution of $\oplus M_{i}$. Now, since the cohomology functors verify that $H_{n}\left(\oplus \mathscr{F}^{i}\right)$ is isomorphic to $\oplus H_{n}\left(\mathscr{F}^{i}\right)$ for every $n \geq 0$ and the tensor product preserves arbitrary direct sums it follows that these right derived functors of the tensor product preserve arbitrary direct sums.

We start by giving a characterization of Gorenstein flat modules over coherent rings.

Proposition 2.1. The following statements are equivalent for $M \in R$-Mod.

(i) $M$ is Gorenstein flat.

(ii) $\operatorname{Tor}_{n}(E, M)=0 \forall n \geq 1$ for every injective $R$-module $E$, $\operatorname{Tor}^{n}(R, M)=$ $0 \forall n \geq 1$ and $M \rightarrow \operatorname{Tor}^{0}(R, M)$ is an isomorphism.

(iii) $\operatorname{Tor}_{n}(E, M)=0 \forall n \geq 1$ for every injective $R$-module $E$, $\operatorname{Tor}^{n}(P, M)=$ $0 \forall n \geq 1$ and every projective $R$-module $P$ and $P \otimes M \rightarrow \operatorname{Tor}^{0}(P, M)$ is an isomorphism again for every projective $R$-module $P$.

Proof. (i) $\Rightarrow$ (ii) It is clear that $\operatorname{Tor}_{n}(E, M)=0 \forall n \geq 1$ and $E$ any injective. Furthermore since $R$ is right coherent there exists an exact sequence $0 \rightarrow M \rightarrow F^{0} \rightarrow F^{1} \rightarrow \cdots$ which may be used to calculate $\operatorname{Tor}^{n}(R, M) \forall n \geq 0$ and so $\operatorname{Tor}^{n}(R, M)=0 \forall n \geq 1$. Finally $M=\operatorname{Ker}\left(F^{0} \rightarrow\right.$ $\left.F^{1}\right) \cong \operatorname{Ker}\left(R \otimes F^{0} \rightarrow R \otimes F^{1}\right)=\operatorname{Tor}^{0}(R, M)$.

(ii) $\Rightarrow$ (iii) This follows from the fact that every projective is a direct summand of a free $R$-module and the naturality of $P \otimes M \rightarrow \operatorname{Tor}^{0}(P, M)$.

(iii) $\Rightarrow$ (i) If $0 \rightarrow M \rightarrow F^{0} \rightarrow F^{1} \rightarrow \cdots$ is a right Flat-resolution, the fact that $\operatorname{Tor}^{n}(R, M)=0 \forall n \geq 1$ gives that $F^{0} \rightarrow F^{1} \rightarrow \cdots$ is exact. But since

$$
M \cong R \otimes M \rightarrow \operatorname{Tor}^{0}(R, M)=\operatorname{Ker}\left(R \otimes F^{0} \rightarrow R \otimes F^{1}\right)
$$

is an isomorphism, we get that $0 \rightarrow M \rightarrow F^{0} \rightarrow F^{1} \rightarrow \cdots$ is exact. In this way $M$ has an exact complete Flat-resolution. On the other hand $\operatorname{Tor}_{n}(E, M)=$ $0 \forall n \geq 1$ gives that the left part of the resolution remains exact whenever $E \otimes-$ is applied for any injective $E$. The corresponding exactness for the right part of the resolution follows from the balanced situation of $-\otimes-$ by the classes of absolute pure and flat modules (on the left and on the right respectively), cf. [7, Proposition 3.3].

Now we study a class of modules which will be very useful for our purposes. In the sequel we will denote by $\mathscr{C}$ the smallest class of $R$-modules we obtain by the following recursive definition: 
(i) $E(M) \in \mathscr{C}$ for all finitely generated modules $M$.

(ii) If $M \in \mathscr{C}$ and $T \subseteq M$ is a submodule, then $E(M / T) \in \mathscr{C}$.

We easily see that all $E \in \mathscr{C}$ are injective

Lemma 2.2. There exists a cardinal $\mathcal{N}$ such that $\operatorname{Card}(E) \leq \mathcal{N}$ for all $E \in \mathscr{C}$.

Proof. It is clear that if $M$ is a finitely generated $R$-module then $\operatorname{Card}(M) \leq$ $\max \left\{\operatorname{Card}(R), \aleph_{0}\right\}$.

On the other hand, since

$$
R^{\left(M^{+}\right)} \rightarrow M^{+}
$$

is an epimorphism, we get a monomorphism

$$
M^{++} \rightarrow\left(R^{\left(M^{+}\right)}\right)^{+}
$$

But $\left(R^{\left(M^{+}\right)}\right)^{+}$is injective and since $M \subseteq M^{++}$we obtain that $E(M) \subseteq$ $\left(R^{\left(M^{+}\right)}\right)^{+}$, and therefore, if $M$ is finitely generated then

$$
\operatorname{Card}(E(M)) \leq \operatorname{Card}\left(\left(R^{\left(M^{+}\right)}\right)^{+}\right) \leq \operatorname{Card}(R)^{\operatorname{Card}(R)}
$$

Now if $T \subseteq E(M)$ with $M$ finitely generated and we consider $E(E(M) / T)$, by analogous reasoning we get that

$$
\operatorname{Card}(E(E(M) / T)) \leq\left(\operatorname{Card}(R)^{\operatorname{Card}(R)}\right)^{\operatorname{Card}(R)}
$$

If we continue with our inductive process as in the definition of $\mathscr{C}$ we get a sequence of cardinal numbers. So there exists a cardinal $\mathscr{N}$ that is bigger than all the elements in our sequence.

From the preceding lemma we get that there exists a set of representatives of $\mathscr{C}$. This fact will be used later.

LeMma 2.3. $\mathscr{C}$ is closed under finite direct sums.

Proof. By the definition of $\mathscr{C}$, we get a sequence of classes

$$
\mathscr{C}_{0} \subseteq \mathscr{C}_{1} \subseteq \mathscr{C}_{2} \subseteq \cdots
$$

such that $\mathscr{C}=\cup_{i=1}^{\infty} C_{i}$ and where $\mathscr{C}_{0}$ is the class of injective envelopes of finitely generated $R$-modules. It is clear that the direct sum of two any elements in $\mathscr{C}_{0}$ is again in $\mathscr{C}_{0}$. Now let us show that the direct sum of any two elements $E_{1}, E_{2} \in \mathscr{C}_{1}$ is also in $\mathscr{C}_{1}$. 
We have that $E_{i}=E\left(E\left(M_{i}\right) / S_{i}\right)$ where $M_{i}$ are finitely generated and $S_{i} \subseteq E\left(M_{i}\right)$ for $i=1,2$ (if $E_{i} \in \mathscr{C}_{0}$ then $S_{i}=0$ ). Then

$$
E_{1} \oplus E_{2} \cong E\left(\frac{E\left(M_{1}\right)}{S_{1}} \oplus \frac{E\left(M_{2}\right)}{S_{2}}\right) \cong E\left(\frac{E\left(M_{1}\right) \oplus E\left(M_{2}\right)}{S_{1} \oplus S_{2}}\right)
$$

Now since $E\left(M_{1}\right) \oplus E\left(M_{2}\right) \in \mathscr{C}_{0}$ the result follows.

The result now follows by an induction argument.

Proposition 2.4. Let $E$ be an injective $R$-module and $E_{1}, E_{2} \in \mathscr{C}$ be submodules of $E$. Then there exists $E_{3} \in \mathscr{C}$ such that $E_{1}+E_{2} \subseteq E_{3} \subseteq E$.

Proof. Since $E_{1} \subseteq E$, we get that $E=E_{1} \oplus E_{1}^{\prime}$. Let $p: E \rightarrow E_{1}^{\prime}$ be the canonical projection. It follows that $p\left(E_{2}\right) \subseteq E\left(p\left(E_{2}\right)\right) \subseteq E_{1}^{\prime}$. Let now $E_{3}=E_{1} \oplus E\left(p\left(E_{2}\right)\right)$ and the result follows from Lemma 2.3.

COROLlary 2.5. Let $E$ be an injective R-module. Then $E$ may be expressed as the direct limit of a directed system of submodules of $E$ which are in $\mathscr{C}$.

Proof. Let $\left\{E_{i}, \varphi_{i j}\right\}_{i \in I}$ be a directed system of all submodules $E_{i}$ of $E$ which are in $\mathscr{C}$ such that $\varphi_{i j}: E_{i} \rightarrow E_{j}$ are inclusion maps. It is enough to show that $E=\cup_{i \in I} E_{i}$ but this follows from the proposition above and from the fact that $E$ is the direct union of a directed system of finitely generated submodules.

The following result may be found in [11, Lemma 5.3.12] but we restate it here without proof for easy reference since we will use it often in this paper.

Lemma 2.6. Let $M$ and $N$ be $R$-modules. Then there exists a cardinal $\mathcal{N}$ which depends on $\operatorname{Card}(N)$ and $\operatorname{Card}(R)$ such that for any morphism $f$ : $N \rightarrow M$ there exists a pure submodule $S$ of $M$ such that $f(N) \subseteq S$ and $\operatorname{Card}(S) \leq \mathcal{N}$.

Definition 2.7. Let $M$ be an $R$-module and $S$ be a submodule of $M$. We will say that $S$ is a Gorenstein pure submodule if $S$ satisfies the following properties:

(i) $0 \rightarrow \operatorname{Tor}_{n}(E, S) \rightarrow \operatorname{Tor}_{n}(E, M) \rightarrow \operatorname{Tor}_{n}(E, M / S) \rightarrow 0$ is exact for all $n \geq 1$ and for any injective $R$-module $E$.

(ii) $0 \rightarrow \operatorname{Tor}^{n}(P, S) \rightarrow \operatorname{Tor}^{n}(P, M) \rightarrow \operatorname{Tor}^{n}(P, M / S) \rightarrow 0$ is exact for all $n \geq 1$ and for any projective $R$-module $P$.

(iii) $\operatorname{Coker}\left(S \rightarrow \operatorname{Tor}^{0}(R, S)\right) \rightarrow \operatorname{Coker}\left(M \rightarrow \operatorname{Tor}^{0}(R, M)\right)$ is an injection.

The following result is an analogous, in the Gorenstein case, of Lemma 2.6.

Lemma 2.8. Let $M \in R$-Mod and $x \in M$. Then there exists a Gorenstein pure submodule $S$ of $M$ and a cardinal $\mathcal{N}$ such that $x \in S$ and $\operatorname{Card}(S) \leq \mathcal{N}$. 
Proof. We first note that every injective $R$-module can be expressed as the direct limit of a family of injective submodules which are in the class $\mathscr{C}$, and every projective $R$-module is a direct limit of a directed family of finitely generated free $R$-modules. Furthermore, $\operatorname{Tor}_{n}$ and Tor $^{n}$ commute with direct limits and direct sums. Thus set $E=\oplus E_{i}$ where $E_{i}$ run over the set of all representatives of $\mathscr{C}$ and $P=\oplus P_{i}$ where $P_{i}$ run over the set of all representatives of finitely generated free $R$-modules.

Let $M$ be any $R$-module and $x \in M$. Then there exists a pure submodule $S$ of $M$ and a cardinal $\mathcal{N}_{0}$ such that $x \in S$ and $\operatorname{Card}(S) \leq \mathcal{N}$ by Lemma 2.6. Let us consider a flat resolution $\cdots \rightarrow F_{1} \rightarrow F_{0} \rightarrow E \rightarrow 0$ of $E$, and form the following commutative diagram

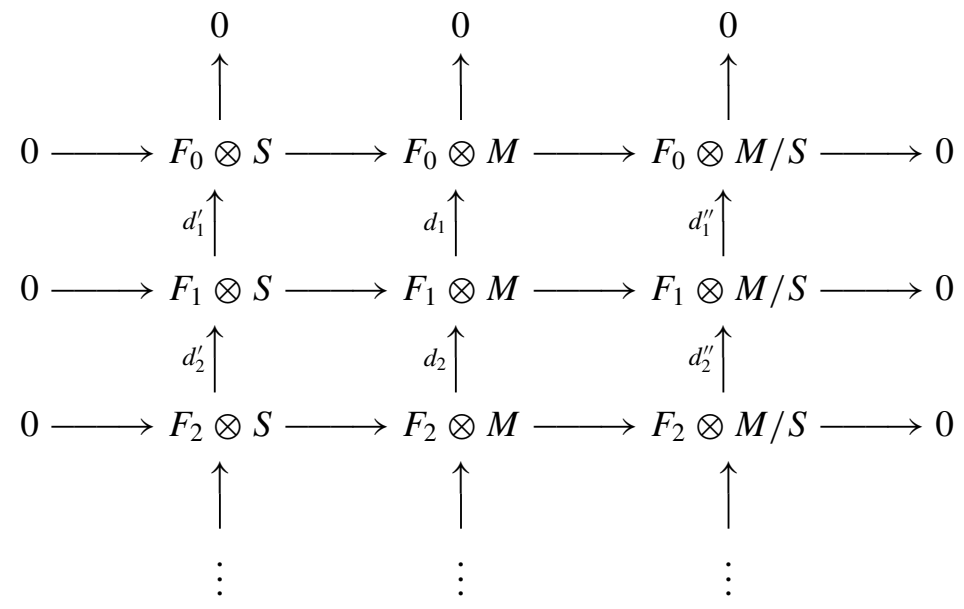

Then we have the exact sequences

$$
\operatorname{Tor}_{n}(E, S) \rightarrow \operatorname{Tor}_{n}(E, M) \rightarrow \operatorname{Tor}_{n}(E, M / S) \forall n \geq 1
$$

and what we want is a submodule $S$ such that the preceding are in fact short exact sequences. Let $n=1$, so we have the exact sequence

$$
\operatorname{Ker}\left(d_{1}^{\prime}\right) / \operatorname{Im}\left(d_{2}^{\prime}\right) \stackrel{f_{1}}{\longrightarrow} \operatorname{Ker}\left(d_{1}\right) / \operatorname{Im}\left(d_{2}\right) \stackrel{g_{1}}{\longrightarrow} \operatorname{Ker}\left(d_{1}^{\prime \prime}\right) / \operatorname{Im}\left(d_{2}^{\prime \prime}\right)
$$

If $f_{1}$ is not injective, there exists $x+\operatorname{Im}\left(d_{2}^{\prime}\right)$ such that $f_{1}\left(x+\operatorname{Im}\left(d_{2}^{\prime}\right)\right) \notin \operatorname{Im}\left(d_{2}\right)$. Then we enlarge $S$ to a pure submodule $P$ of $M$ such that $<f_{1}(x), \operatorname{Im}\left(d_{2}\right)>\subseteq$ $F_{1} \otimes P$ and $\operatorname{Card}(P) \leq \mathcal{N}_{1}$ for certain cardinal $\mathcal{N}_{1}$. Let us consider now $d_{2}\left(F_{2} \otimes\right.$ $P) \subseteq \operatorname{Ker}\left(\left.d_{1}\right|_{F_{1} \otimes P}\right)$. We now enlarge $P$ to $P^{\prime}$ such that $g_{1}\left(\operatorname{Ker}\left(\left.d_{1}\right|_{F_{1} \otimes P}\right) / d_{2}\left(F_{2}\right.\right.$ $\otimes P)) \subseteq F_{2} \otimes\left(M / P^{\prime}\right)$ and such that $\operatorname{Card}\left(P^{\prime}\right) \leq \mathcal{N}_{2}$ for certain $\mathcal{N}_{2}$. Then consider an analogous diagram as above associated to $0 \rightarrow P^{\prime} \rightarrow M \rightarrow$ $M / P^{\prime} \rightarrow 0$ and get a sequence as $(*)$. If the new $g_{1}$ is not onto, then there 
exists $\bar{z} \in \operatorname{Ker}\left(d_{1}^{\prime \prime}\right) / \operatorname{Im}\left(d_{2}^{\prime \prime}\right)$ which is not in $\operatorname{Im}\left(g_{1}\right)$. Then we may consider $P^{\prime \prime} \subseteq M$ such that $P^{\prime} \subseteq P^{\prime \prime}, \operatorname{Card}\left(P^{\prime \prime}\right) \leq \mathscr{N}_{3}$ for a cardinal $\mathscr{N}_{3}$ and there is $x \in F_{1} \otimes P^{\prime \prime}$ such that $d_{1}(x)=z$. Now we consider the exact sequence $0 \rightarrow P^{\prime \prime} \rightarrow M \rightarrow M / P^{\prime \prime} \rightarrow 0$ and its sequence of the form $(*)$ and reason as above with the new $f_{1}$.

Now let $S_{1}$ be the module obtained with the "limit" of this procedure, whose cardinal is bounded by another certain cardinal, which is bigger than all the cardinals we got during the procedure and which satisfies that

$$
0 \rightarrow \operatorname{Tor}_{1}\left(E, S_{1}\right) \rightarrow \operatorname{Tor}_{1}(E, M) \rightarrow \operatorname{Tor}_{1}\left(E, M / S_{1}\right) \rightarrow 0
$$

is exact. Then, from this, we apply the same reasoning to get a new $S_{2}$ such that

$$
0 \rightarrow \operatorname{Tor}_{2}\left(E, S_{2}\right) \rightarrow \operatorname{Tor}_{2}(E, M) \rightarrow \operatorname{Tor}_{2}\left(E, M / S_{2}\right) \rightarrow 0
$$

is exact. But it is possible that we may loose the exactness of the Tor ${ }_{1}$ 's sequence. So we get from $S_{2}$ a new submodule $S_{3}$ such that the Tor ${ }_{1}$ 's sequence is exact. In this way, we construct $S_{i}$ such that the Tor $_{n}$ 's sequences are exact in a new zig-zag process, going back $n$ steps and going forward $n+1$. Now let $S^{\prime}$ be the union of all the $S_{i}$ that we get. It is clear that $S^{\prime}$ is such that

$$
0 \rightarrow \operatorname{Tor}_{1}\left(E, S^{\prime}\right) \rightarrow \operatorname{Tor}_{1}(E, M) \rightarrow \operatorname{Tor}_{1}\left(E, M / S^{\prime}\right) \rightarrow 0
$$

is exact and that there is a cardinal $\mathscr{N}^{\prime}$ such that $\operatorname{Card}\left(S^{\prime}\right) \leq \mathscr{N}^{\prime}$.

For the second step in the proof, we need a module $S^{\prime \prime}$ which verifies that

$$
0 \rightarrow \operatorname{Tor}^{n}\left(P, S^{\prime \prime}\right) \rightarrow \operatorname{Tor}^{n}(P, M) \rightarrow \operatorname{Tor}^{n}\left(P, M / S^{\prime \prime}\right) \rightarrow 0 \forall n \geq 1
$$

But $S^{\prime \prime}$ may be obtained from $S^{\prime}$ by following an analogous reasoning as we followed to get $S^{\prime}$, using a right Flat-resolution of $P$, and again $\operatorname{Card}\left(S^{\prime \prime}\right)$ is bounded by certain cardinal $\mathscr{N}^{\prime \prime}$.

Finally we have to find a new module $S^{\prime \prime \prime}$ from $S^{\prime \prime}$ such that

$$
\operatorname{Coker}\left(S^{\prime \prime \prime} \rightarrow \operatorname{Tor}^{0}\left(R, S^{\prime \prime \prime}\right)\right) \stackrel{f}{\longrightarrow} \operatorname{Coker}\left(M \stackrel{h_{M}}{\longrightarrow} \operatorname{Tor}^{0}(R, M)\right)
$$

is an injection. So if there is $z \in \operatorname{Coker}\left(S^{\prime \prime} \rightarrow \operatorname{Tor}^{0}\left(R, S^{\prime \prime}\right)\right)$ such that $f(z)=$ $\sum_{i=1}^{n}\left(x_{i} \otimes y_{i}\right) \in h_{M}(M)$ we may get a finitely generated submodule $T$ of $M$ such that each $y_{i} \in T$. So we can consider $S^{\prime \prime \prime}=S^{\prime \prime}+T$. We note that in this way we can get $S_{0} \subseteq M$ whose cardinal is less than or equal to the cardinal of $S^{\prime \prime}$ and such that it contains all the generators in $M$ of those elements which go to zero. So let $S^{\prime \prime \prime}=S^{\prime \prime}+S_{0}$.

Now we get a module which satisfies the third condition, but it is possible that after the procedure we no longer have that our module verifies the first or 
the second conditions, or both of them. So if we call the module we get after this procedure of three steps $S_{1}$, we have to enlarge $S_{1}$ to $S_{2}$ applying again our three steps. Now let $S=\cup_{i=1}^{\infty} S_{i}$, the union of all the modules we get in each procedure of three steps. Then $S$ is the desired module.

Proposition 2.9. Let $M$ be an $R$-module and $S$ be a submodule of $M$. If $M / S$ is Gorenstein flat, then $S \subseteq M$ is Gorenstein pure.

Proof. Since $M / S$ is Gorenstein flat, we have that the sequence

$0=\operatorname{Tor}_{n+1}(E, M / S) \rightarrow \operatorname{Tor}_{n}(E, S) \rightarrow \operatorname{Tor}_{n}(E, M) \rightarrow \operatorname{Tor}_{n}(E, M / S)=0$

is exact for all injectives $E$ and for all $n \geq 1$. Similarly, we have that the sequence

$$
0=\operatorname{Tor}^{n}(P, M / S) \rightarrow \operatorname{Tor}^{n}(P, S) \rightarrow \operatorname{Tor}^{n}(P, M) \rightarrow \operatorname{Tor}^{n+1}(P, M / S)=0
$$

is exact for all projectives $P$ and for all $n \geq 1$.

Finally, we consider the following commutative diagram

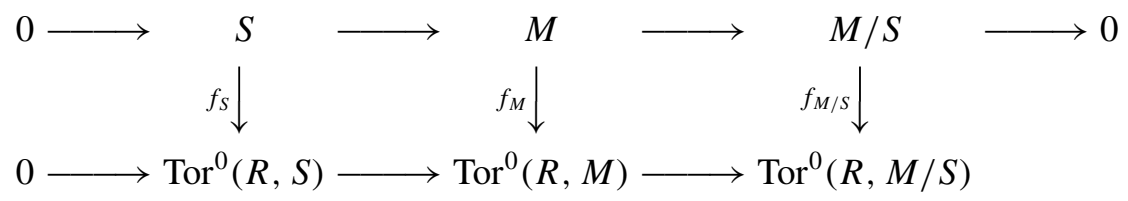

with exact rows. But $f_{M / S}$ is an isomorphism since $M / S$ is Gorenstein flat. So by the Snake Lemma, $\operatorname{Coker}\left(f_{S}\right) \rightarrow \operatorname{Coker}\left(f_{M}\right)$ is an isomorphism and thus we are done.

Proposition 2.10. Let $M$ be an $R$-module and $S$ a Gorenstein pure submodule of $M$. If $M$ is Gorenstein flat, then $S$ and $M / S$ are also Gorenstein flat.

Proof. By the definition of Gorenstein pure it is clear that if $M$ is Gorenstein flat then $\operatorname{Tor}_{n}(E, S)=\operatorname{Tor}_{n}(E, M / S)=0 \forall n \geq 1$ and for every injective $R$-module $E$ and $\operatorname{Tor}^{n}(R, S)=\operatorname{Tor}^{n}(R, M / S)=0 \forall n \geq 1$. On the other hand

$$
\operatorname{Coker}\left(S \rightarrow \operatorname{Tor}^{0}(R, S)\right) \rightarrow \operatorname{Coker}\left(M \rightarrow \operatorname{Tor}^{0}(R, M)\right)
$$

is an injection, that is, if we consider the diagram

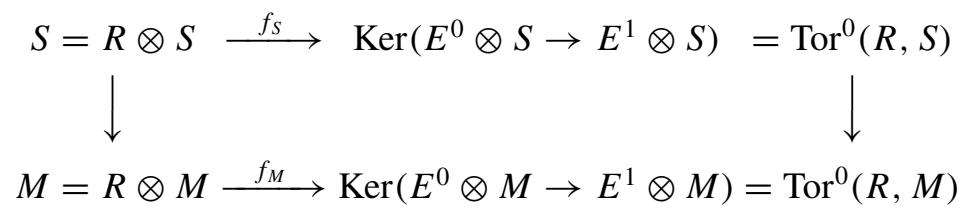


and if $y \in \operatorname{Tor}^{0}(R, S)$ is such that its image $z \in \operatorname{Tor}^{0}(R, M)$ is in $\operatorname{Im}\left(f_{M}\right)$, then $y \in \operatorname{Im}\left(f_{S}\right)$. Therefore if $M \rightarrow \operatorname{Tor}^{0}(R, M)$ is an isomorphism, by the commutativity of the diagram, $S \rightarrow \operatorname{Tor}^{0}(R, S)$ is a monomorphism and by the preceding is also onto. Thus $S$ is Gorenstein flat. Finally, let us consider the commutative diagram

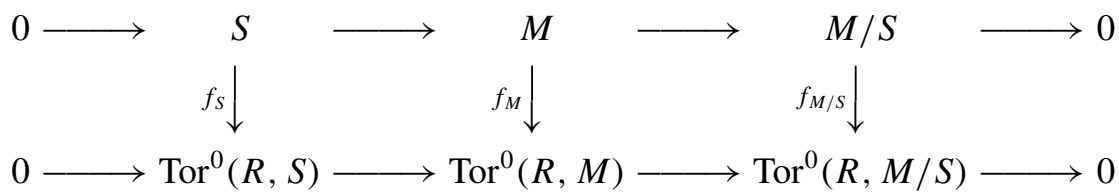

If we apply the Snake Lemma, since $f_{S}$ and $f_{M}$ are isomorphisms, then $f_{M / S}$ is also an isomorphism and so, $M / S$ is Gorenstein flat.

THEOREM 2.11. Let $R$ be a right coherent ring and let $\mathscr{F}$ denote the class of Gorenstein flat left $R$-modules. Then $\left(\mathscr{F}, \mathscr{F}^{\perp}\right)$ is a complete cotorsion theory.

Proof. By Lemma 2.8 and Proposition 2.10, if $F \in \mathscr{F}$ then $F$ can be written as the direct union of a continuous chain of submodules $\left(F_{\alpha}\right)_{\alpha<\lambda}$ with $\lambda$ an ordinal number such that $F_{0} \in \mathscr{F}, F_{\alpha+1} / F_{\alpha} \in \mathscr{F}$ when $\alpha+1<\lambda$ with $\operatorname{Card}\left(F_{0}\right), \operatorname{Card}\left(F_{\alpha+1} / F_{\alpha}\right) \leq \mathcal{N}$. Therefore if $B$ is the direct sum of all representatives of $\mathscr{F}$ such that their cardinal is less than or equal to $\mathcal{N}$, then $M \in \mathscr{F} \perp$ if and only if $\operatorname{Ext}_{R}^{1}(B, M)=0$.

Let now $N$ be any $R$-module. We will use the procedure in [5, Theorem 10] to get an exact sequence $0 \rightarrow N \rightarrow A \rightarrow F \rightarrow 0$ such that $A \in \mathscr{F}^{\perp}$ and $F \in \mathscr{F}$.

Let $B$ be an $R$-module and $0 \rightarrow S \rightarrow P \rightarrow B \rightarrow 0$ be exact with $P$ projective. For any ordinal $\lambda$, by transfinite induction we can construct a continuous chain of submodules $\left(N_{\alpha}\right)_{\alpha<\lambda}$ such that $N_{0}=N$ and if $\alpha+1<\lambda$, any morphism $S \rightarrow N_{\alpha}$ has an extension $P \rightarrow N_{\alpha+1}$. Then each $N_{\alpha+1} / N_{\alpha}$ is a direct sum of copies of $P / S \cong B$ for $\alpha+1<\lambda$. We now use [11, Corollary 7.3.2] with $S$ the set of that Corollary to find a corresponding ordinal $\lambda$ and define $A=\cup_{\alpha<\lambda} N_{\alpha}$ and $F=A / N$. Then for any morphism $S \rightarrow A$ there is a factorization $S \rightarrow N_{\alpha} \rightarrow A$ for some $\alpha<\lambda$. Since $\lambda$ is a limit ordinal, we have $\alpha+1<\lambda$ and so, by construction there is a morphism $P \rightarrow N_{\alpha+1}$ which agrees with $S \rightarrow N_{\alpha}$. But this is just to say that $A \in \mathscr{F} \perp$. If we put $F_{\alpha}=N_{\alpha} / N$ we get that $F_{0}$ and $F_{\alpha+1} / F_{\alpha}$ are in $\mathscr{F}$. Now using an easy induction over the exact sequence of Tor's associated with the exact sequence $0 \rightarrow F_{\alpha} \rightarrow F_{\alpha+1} \rightarrow F_{\alpha+1} / F_{\alpha} \rightarrow 0$ we get that $F_{\alpha} \in \mathscr{F}$ for each $\alpha<\lambda$. Since $\mathscr{F}$ is closed under direct limits, it follows that $F \in \mathscr{F}$.

If $M$ is any $R$-module and $0 \rightarrow K \rightarrow P \rightarrow M \rightarrow 0$ is a projective presentation of $M$ and if we apply the preceding to $K$, we get an exact sequence 
$0 \rightarrow K \rightarrow A \rightarrow F \rightarrow 0$ with $A \in \mathscr{F}^{\perp}$ and $F \in \mathscr{F}$. Then let us consider the pushout diagram:

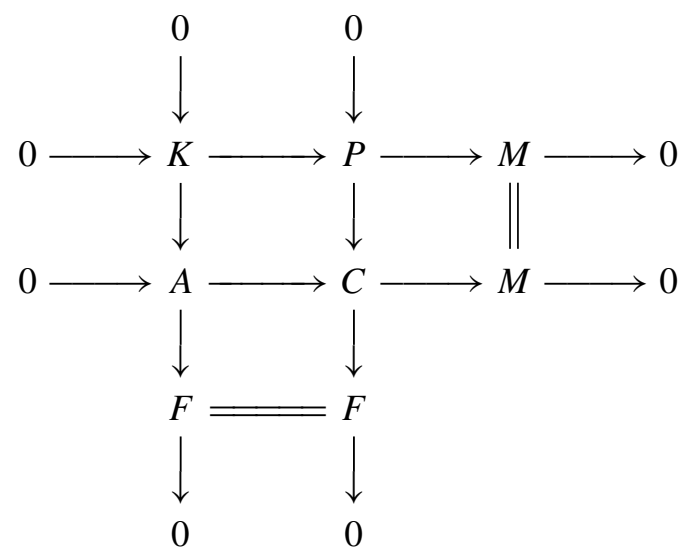

Since $P, F \in \mathscr{F}$ we get that $C \in \mathscr{F}$. Suppose now that $M \in^{\perp}\left(\mathscr{F}^{\perp}\right)$. Since $A \in \mathscr{F}^{\perp}$, then the central row splits and so $M \in \mathscr{F}$. This gives us that $\mathscr{F}=$ ${ }^{\perp}\left(\mathscr{F}^{\perp}\right)$, and therefore $\left(\mathscr{F}, \mathscr{F}^{\perp}\right)$ is a cotorsion theory with enough injectives and projectives. The fact that $\left(\mathscr{F}, \mathscr{F}^{\perp}\right)$ is hereditary is immediate from the definition of $\mathscr{F}$.

Now we are able to prove the following important theorem.

THEOREM 2.12. Let $R$ be a right coherent ring and $\mathscr{F}$ denote the class of Gorenstein flat left $R$-modules. Then $\left(\mathscr{F}, \mathscr{F}^{\perp}\right)$ is a hereditary perfect cotorsion theory.

Proof. Let us show first that $\left(\mathscr{F}, \mathscr{F}^{\perp}\right)$ is hereditary. So let us consider an exact sequence $0 \rightarrow F^{\prime} \rightarrow F \rightarrow F^{\prime \prime} \rightarrow 0$ where $F$ and $F^{\prime \prime}$ are Gorenstein flat. The Snake Lemma applied to the diagram

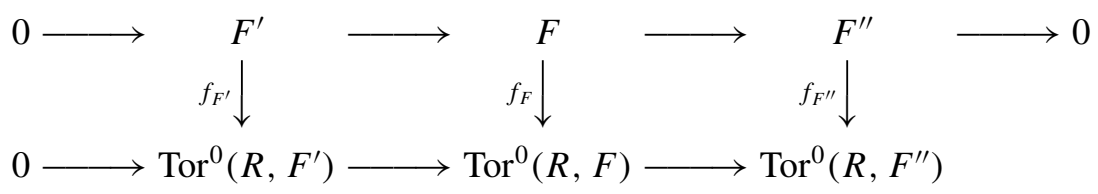

shows that $f_{F^{\prime}}$ is an isomorphism and that

$$
\operatorname{Tor}^{0}(R, F) \rightarrow \operatorname{Tor}^{0}\left(R, F^{\prime \prime}\right) \rightarrow 0
$$

is exact. Since $F$ and $F^{\prime \prime}$ are Gorenstein flat and we have the exact sequence

$$
0 \rightarrow \operatorname{Tor}^{0}\left(R, F^{\prime}\right) \rightarrow \operatorname{Tor}^{0}(R, F) \rightarrow \operatorname{Tor}^{0}\left(R, F^{\prime \prime}\right) \rightarrow \cdots
$$


with $\operatorname{Tor}^{0}(R, F) \rightarrow \operatorname{Tor}^{0}\left(R, F^{\prime \prime}\right) \rightarrow 0$ exact, we get that

$$
0 \rightarrow \operatorname{Tor}^{1}\left(R, F^{\prime}\right) \rightarrow \operatorname{Tor}^{1}(R, F) \rightarrow \operatorname{Tor}^{1}\left(R, F^{\prime \prime}\right) \rightarrow \cdots
$$

is exact which implies that $\operatorname{Tor}^{n}\left(R, F^{\prime}\right)=0 \forall n \geq 1$. Moreover it is easy to show also that $\operatorname{Tor}_{n}\left(E, F^{\prime}\right)=0 \forall n \geq 1$, and therefore $\left(\mathscr{F}, \mathscr{F}^{\perp}\right)$ is hereditary.

Finally, since $\left(\mathscr{F}, \mathscr{F}^{\perp}\right)$ is complete and $\mathscr{F}$ is closed under direct limits we get that $\left(\mathscr{F}, \mathscr{F}^{\perp}\right)$ is perfect by [11, Theorem 7.2.6].

H. Holm in [12, Theorem 3.9] proved that if $R$ is commutative and noetherian then the class $\mathscr{F}$ of Gorenstein flat modules is "projectively resolving" (i.e. if $0 \rightarrow F^{\prime} \rightarrow F \rightarrow F^{\prime \prime} \rightarrow 0$ is an exact sequence of modules with $F \in \mathscr{F}$ then $F^{\prime} \in \mathscr{F}$ if and only if $F \in \mathscr{F}$ ). So this is an extension of Holm's result.

\section{Modules have weakly Gorenstein flat covers}

The purpose of this section is to find a perfect cotorsion theory which will give us the existence of weakly Gorenstein flat covers for any $R$-module.

In section 3 we studied Gorenstein flat modules. Now we will consider a class $\mathscr{F}$ of modules larger than the class of Gorenstein flat modules.

Let now $\mathscr{F}$ be the class of left $R$-modules $M$ such that there exists an exact sequence

$$
\cdots \rightarrow F_{1} \rightarrow F_{0} \rightarrow M \rightarrow 0
$$

of flat left $R$-modules which remains exact whenever $E \otimes_{R}-$ is applied to it for every injective right $R$-module $E$. We note that $\mathscr{F}$ may be characterized also by $M \in \mathscr{F}$ if and only if $\operatorname{Tor}_{i}(E, M)=0$ for all $i \geq 1$ and for any injective $R$-module $E$. Now following the terminology of [4] we will call the modules in $\mathscr{F}$ weakly Gorenstein flat modules.

Let $\mathscr{C}$ be the same class of modules of Section 2. The following result is analogous to [11, Lemma 7.4.5].

Proposition 3.1. Let $M \in \mathscr{F}$. If $x \in M$, then there exists a cardinal $\mathcal{N}$ and a submodule $S$ of $M$ with $x \in S$ such that $S, M / S \in \mathscr{F}$ and $\operatorname{Card}(S) \leq \mathcal{N}$.

Proof. We will reason as in Lemma 2.8, constructing the submodule $S$ in two steps: first we will construct an exact complex of flat left $R$-modules and second we will use this complex to find another complex of flat modules such that $E \otimes_{R}-$ makes it exact.

So let $\cdots \rightarrow F_{2} \stackrel{d_{2}}{\longrightarrow} F_{1} \stackrel{d_{1}}{\longrightarrow} F_{0} \stackrel{f}{\longrightarrow} M \rightarrow 0$ be the corresponding exact flat resolution for $M$ and let $x \in M$. Now let $y \in F_{0}$ be such that $y=f(x)$ and consider the inclusion $\langle y\rangle \rightarrow F_{0}$. Then by Lemma 2.6, there is a cardinal $\mathcal{N}_{0}$ and a pure submodule $S_{0} \subseteq F_{0}$ such that $\langle y\rangle \subseteq S_{0}$ and $\operatorname{Card}\left(S_{0}\right) \leq$ 
$\mathcal{N}_{0}$. Now consider $S_{0} \cap \operatorname{Ker}(f) \subseteq M$. Then there exists $D_{1} \subseteq F_{1}$ such that $d_{1}\left(D_{1}\right)=S_{0} \cap \operatorname{Ker}(f)$. Again, by Lemma 2.6, there is a pure submodule $S_{1}$ in $F_{1}$ and a cardinal $\mathscr{N}_{1}$ such that $D_{1} \subseteq S_{1}$ and $\operatorname{Card}\left(S_{1}\right) \leq \mathscr{N}_{1}$. Now consider $d_{1}\left(S_{1}\right) \subseteq F_{0}$. Then there exists a pure submodule $S_{2}$ in $F_{0}$ and a cardinal $\mathcal{N}_{2}$ such that $d_{1}\left(S_{1}\right) \subseteq S_{2}$ and $\operatorname{Card}\left(S_{2}\right) \leq \mathcal{N}_{2}$. We now consider $f\left(S_{2}\right)$.

We start the construction again going back by considering $S_{2} \cap \operatorname{Ker}(f)$ and proceeding as before, going $n$ steps forward, going back $n+1$ steps and $n+1$ forward again.

Then we take the union of all the complexes constructed in the "zig-zag" process

$$
S^{*}=\cdots \rightarrow S_{1} \rightarrow S_{0} \rightarrow S \rightarrow 0
$$

where $S=f\left(S_{0}\right) \subseteq M$, which contains the element $x$ and that by the construction, there exists a cardinal $\mathcal{N}$ such that $\operatorname{Card}(S) \leq \mathcal{N}$. The previous complex is exact by its construction and it is formed by flat modules since all of them are pure submodules of flat modules.

In the second step of the proof, our objective is to construct $S \subseteq M$ with an exact sequence as before and such that it remains exact when $E \otimes_{R}-$ is applied to it for every injective $R$-module $E$. By Corollary 2.5 we know that every injective module $E$ may be expressed as the direct limit of a family of injective submodules which are in $\mathscr{C}$. Then we consider the module $I=\oplus E_{i}$ where $E_{i}$ runs over the set of representatives of $\mathscr{C}$. Now if a sequence is such that $I \otimes_{R}$ - leaves it exact, by the commutativity of the tensor products with direct sums we get that $E_{i} \otimes_{R}-$ will also leave the sequence exact and from the commutativity of direct limits and the tensor products our sequence will remain exact under $E \otimes_{R}$ - for every injective module $E$.

Then let $I$ be the preceding module and let us consider the complex

$$
\cdots \stackrel{d_{2}^{\prime}}{\longrightarrow} I \otimes S_{1} \stackrel{d_{1}^{\prime}}{\longrightarrow} I \otimes S_{0} \stackrel{f^{\prime}}{\longrightarrow} I \otimes S \rightarrow 0
$$

This complex is a subcomplex of

$$
\cdots \stackrel{d_{2}}{\longrightarrow} I \otimes F_{1} \stackrel{d_{1}}{\longrightarrow} I \otimes F_{0} \stackrel{f}{\longrightarrow} I \otimes M \rightarrow 0
$$

which is exact since $M \in \mathscr{F}$ and $I$ is a direct sum of injectives. Suppose then without lost of generality that $\operatorname{Ker}\left(f^{\prime}\right) \neq \operatorname{Im}\left(d_{1}^{\prime}\right)$. Then there is a pure submodule $S_{1}^{\prime}$ of $F_{1}$ and a cardinal $\mathscr{N}_{1}$ such that $S_{1} \subseteq S_{1}^{\prime}, \operatorname{Ker}\left(f^{\prime}\right) \subseteq \operatorname{Im}\left(\left.d_{1}\right|_{I \otimes S_{1}^{\prime}}\right)$ and $\operatorname{Card}\left(S_{1}^{\prime}\right) \leq \mathcal{N}_{1}$. Now let $S^{\prime}$ be the image of $S_{1}^{\prime}$ under the morphism $F_{1} \rightarrow F_{0}$, and let $S_{0}^{\prime}$ be a pure submodule of $F_{0}$ and $\mathcal{N}_{2}$ be a cardinal such that $S^{\prime} \subseteq S_{0}^{\prime}$, $\operatorname{Im}\left(\left.d_{1}\right|_{I \otimes S_{1}^{\prime}}\right) \subseteq I \otimes S_{0}^{\prime}$, and $\operatorname{Card}\left(S_{0}^{\prime}\right) \leq \mathcal{N}_{2}$. Then let $S^{\prime}$ be the image of $S_{0}^{\prime}$ under the morphism $F_{0} \rightarrow M$. Then we go back again and start another "zigzag" process with $\operatorname{Ker}\left(\left.f\right|_{I \otimes S_{0}^{\prime}}\right)$ and $\operatorname{Im}\left(\left.d_{1}\right|_{I \otimes S_{1}^{\prime}}\right)$. We consider the union of all 
the complexes we get in this last "zig-zag" process

$$
T^{*}=\cdots \rightarrow T_{1} \rightarrow T_{0} \rightarrow T \rightarrow 0
$$

By the construction above, there is a cardinal $\mathscr{N}$ such that $\operatorname{Card}(T) \leq \mathscr{N}$ and when $E \otimes_{R}$ - is applied to the complex $T^{*}$ we get an exact complex. But $T^{*}$ may not be exact. So we apply again the "zig-zag" process we used to get $S^{*}$ and get an exact complex but which may not remain exact when $E \otimes_{R}-$ is applied. So again we use the same reasoning we used to get $T^{*}$ and obtain a new complex. The "limit" over these two procedures gives us a module $S$, a cardinal $\mathcal{N}$, and a complex $S^{*}$ as we desired. Furthermore, $M / S$ is also in $\mathscr{F}$ since the quotient complex $F^{*} / S^{*}$ is exact and it remains exact when $E \otimes_{R}-$ is applied to it since $F^{*}$ and $S^{*}$ satisfy the two conditions.

Now reasoning analogously as in Theorem 2.11 and using the previous Lemma we get the following

THEOREM 3.2. $\left(\mathscr{F}, \mathscr{F}^{\perp}\right)$ is a complete hereditary cotorsion theory in $R$ Mod.

COROLlaRY 3.3. $\left(\mathscr{F}, \mathscr{F}^{\perp}\right)$ is a perfect hereditary cotorsion theory in $R$ Mod.

Proof. By the Theorem above, every module has an $\mathscr{F}$-precover and $\mathscr{F}^{\perp}$ preenvelope and therefore the result follows from [11, Theorem 7.2.6] since it is clear that $\mathscr{F}$ is closed under direct limits.

As first examples we note that if $F$ is a flat $R$-module, then the $\mathscr{F}$-cover of $F$ is $F \stackrel{i d}{\longrightarrow} F$. If $R$ has finite global dimension, then $\mathscr{F}$ is the class of flat modules and so $\mathscr{F}$-covers are in fact flat covers whose existence was shown in [2]. Finally, if $R$ is a Gorenstein ring, then $\mathscr{F}$ becomes the class of Gorenstein flat modules (cf. [8]), and so, the $\mathscr{F}$-cover of an $R$-module is the Gorenstein flat cover whose existence was shown in [9].

As a final remark we note that Theorem 2.11 can also be proved by showing that for every Gorenstein flat $R$-module $F$ and $x \in F$, there exists a submodule $S$ of $F$ and a cardinal $\mathcal{N}$ such that $x \in S, \operatorname{Card}(S) \leq \mathcal{N}$ and $S$ and $F / S$ are Gorenstein flat. This can be proved using the same "zig-zag" argument in Proposition 3.1 over the exact flat resolution on both sides of $F$ going $n$ steps on the right and $n+1$ on the left. But the result obtained in Lemma 2.8 is more general.

ACKNOwLEDgements. This paper was written while the third author was visiting University of Lexington, Kentucky supported by Ministerio de Educacion y Cultura grant EX 00 34845300. The third author is also partially supported by DGES grant PB98-1005 and Junta de Andalucía FQM 0211 


\section{REFERENCES}

1. Auslander, M., Anneaux de Gorenstein et torsion en algèbre commutative, Séminaire d'algèbre commutative 1966/67, notes by M. Mangeney, C. Peskine and L. Szpiro, École Normale Supérieure de Jeunes Filles, Paris (1967).

2. Belshoff, R., Enochs, E., and Xu, J., The existence of flat covers, Proc. Amer. Math. Soc. 122 (1994), 985-991.

3. Bican, L., El Bashir, R., and Enochs, E. E., All Modules have flat covers, Bull. London Math. Soc. 33 (2001), 385-390.

4. Christensen, L. W., Gorenstein Dimensions, Lecture Notes in Math. 1747 (2000).

5. Eklof, P., and Trlifaj, J., How to make Ext vanish, Bull. London Math. Soc. 33(4) no. 1 (2001), 41-51.

6. Enochs, E., Injective and flat covers, envelopes and resolvents, Israel J. Math. 39(3) (1981), 189-209.

7. Enochs, E., and Jenda, O. M. G., Balanced Functors Applied to Modules, J. Algebra 92 (1985), 303-310.

8. Enochs, E. E., Jenda, O. M. G., and Torrecillas, B., Gorenstein flat modules, Nanjing Daxue Xuebao Bannian Kan 10 (1993), 1-9.

9. Enochs, E. E., and Xu, J., Gorenstein flat covers of modules over Gorenstein rings, J. Algebra 181 (1996), 288-313.

10. Enochs, E. E., Jenda, O. M. G., and Xu, J., Foxby duality and Gorenstein injective and projective modules, Trans. Amer. Math. Soc. 348(8) (1996), 3223-3234.

11. Enochs, E. E., and Jenda, O. M. G., Relative Homological Algebra, De Gruyter, Berlin-New York (2000).

12. Holm, H., Gorenstein Homological Dimensions, preprint.

13. Hovey, M., Cotorsion Theories, Model Category Structures and Representation Theory, preprint.

14. Salce, L., Cotorsion theories for abelian groups, Sympos. Math. Vol. XXIII, pp. 11-32 (1979).

15. Xu, J., Flat covers of modules, Lecture Notes in Math. 1634 (1996).

DEPARTMENT OF MATHEMATICS

UNIVERSITY OF KENTUCKY

LEXINGTON, KY 40506

USA

E-mail: enochs@ms.uky.edu
DEPARTMENT OF MATHEMATICS

AUBURN UNIVERSITY

AUBURN, ALABAMA 36849-5307

USA

E-mail: jendaov@mail.auburn.edu

DEPARTMENT OF ALGEBRA AND ANALYSIS

UNIVERSITY OF ALMERIA

ALMERIA 04120

SPAIN

E-mail: jlopez@ual.es 